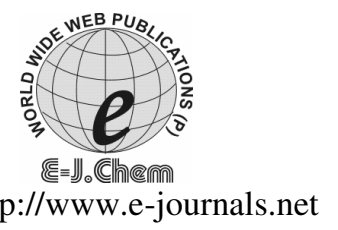

ISSN: 0973-4945; CODEN ECJHAO

E-Journal of Chemistry

2010, 7(4), 1514-1522

\title{
Development and Validation of a Precise and Stability Indicating LC Method for the Determination of Benzalkonium Chloride in Pharmaceutical Formulation Using an Experimental Design
}

\author{
HARSHAL K. TRIVEDI* and MUKESH C. PATEL \\ *Analytical Research Lab, Cadila Pharmaceutical Ltd, Dholka, Gujarat, India. \\ P.S. Science and H.D.Patel Arts College, Kadi, Gujarat, India. \\ trivedi_harshal@rediffmail.com
}

Received 3 June 2009; Accepted 1 August 2009

\begin{abstract}
A simple, precise, shorter runtime and stability indicating reversephase high performance liquid chromatographic method has been developed and validated for the quantification of benzalkonium chloride (BKC) preservative in pharmaceutical formulation of sparfloxacin eye drop. The method was successfully applied for determination of benzalkonium chloride in various ophthalmic formulations like latanoprost, timolol, dexametasone, gatifloxacin, norfloxacin, combination of moxifloxacin and dexamethasone, combination of nepthazoline $\mathrm{HCl}$, zinc sulphate and chlorpheniramine maleate, combination of tobaramycin and dexamethasone, combination of phenylephrine $\mathrm{HCl}$, naphazoline $\mathrm{HCl}$, menthol and camphor. The RP-LC separation was achieved on an Purospher Star RP-18e $75 \mathrm{~mm} \times 4.0 \mathrm{~mm}, 3.0 \mu$ in the isocratic mode using buffer: acetonitrile (35: $65, \mathrm{v} / \mathrm{v})$, as the mobile phase at a flow rate of $1.8 \mathrm{~mL} / \mathrm{min}$. The methods were performed at $215 \mathrm{~nm}$; in LC method, quantification was achieved with PDA detection over the concentration range of 50 to $150 \mu \mathrm{g} / \mathrm{mL}$. The method is effective to separate four homologs with good resolution in presence of excipients, sparfloxacin and degradable compound due to sparfloxacin and BKC within five minutes. The method was validated and the results were compared statistically. They were found to be simple, accurate, precise and specific. The proposed method was validated in terms of specificity, precision, recovery, solution stability, linearity and range. All the validation parameters were within the acceptance range and concordant to ICH guidelines.
\end{abstract}

Keywords: Benzalkonium chloride, Sparfloxacin, Stability indicating, Liquid chromatography, Ophthalmic solution. 


\section{Introduction}

The determination of low concentration preservative in pharmaceutical formulation constitutes a challenging problem in current pharmaceutical analysis. Ophthalmic solutions containing the pharmaceutical association between active pharmaceutical ingredient sparfloxacin and preservative BKC.

Sparfloxacin eye drops contain sparfloxacin, a synthetic broad-spectrum antimicrobial agent for ophthalmic solution administration. Commercially available sparfloxacin ophthalmic solution is a clear, yellow colored solution of the drug in sterile water for injection; benzalkonium chloride is added as a preservative.

Benzalkonium chloride (Figure 1) is a mixture of alkyls, including all or some of the group beginning with $n-\mathrm{C}_{8} \mathrm{H}_{17}$ and extending through higher homologs ${ }^{1}$, with $n-\mathrm{C}_{12} \mathrm{H}_{25}$, $n-\mathrm{C}_{14} \mathrm{H}_{29}$ and $n-\mathrm{C}_{16} \mathrm{H}_{33}$ comprising the major portion. Benzalkonium chloride ${ }^{2}$ solutions are rapidly acting biocidal agents with a moderately long duration of action. They are active against bacteria and some viruses, fungi and protozoa. Its use as a preservative in cosmetic suches eye and nasal drops attests to its general safety.

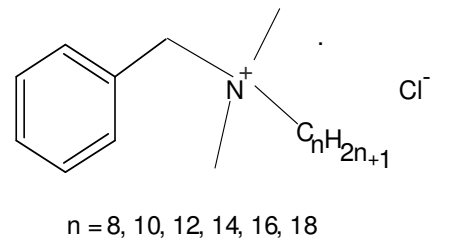<smiles>CCCCN(C)[C+]c1ccccc1</smiles>

$\left[\mathrm{C}_{6} \mathrm{H}_{5} \mathrm{CH}_{2} \mathrm{~N}\left(\mathrm{CH}_{3}\right)_{2} \mathrm{R}\right] \mathrm{Cl}$

Figure 1. Structure of benzalkonium chloride (BKC)

This paper portraits a simple, precise and accurate liquid chromatographic method for quantification of BKC in sparfloxacin ophthalmic solution. A comprehensive literature search revealed that review of preparation, pharmacology ${ }^{3}$, structural relationship, effect with intended use $^{3}$, HPLC $^{4,5}$, LC-MS ${ }^{5}$, MS, electrophoresis ${ }^{6}$ and other chromatographic methods for the determination of sparfloxacin and $\mathrm{BKC}$ were reported as a long run time and less resolution for analysis of BKC, as such there is lack of a suitable procedure for the quantification and estimation of BKC preservative in pharmaceutical formulation of sparfloxacin. Therefore, the aim of this work is the development ${ }^{7} \&$ validation $^{8}$ of a simple, precise accurate and specific method for quantification of benzalkonium chloride (BKC) by HPLC-UV detection, in pharmaceutical formulation of sparfloxacin eye drops.

\section{Experimental}

HPLC grade acetonitrile, potassium dihydrogen orthophosphate, 1- octane sulphonate and sodium hydroxide were used to prepare the mobile phase and were purchased from SigmaAldrich (Milan, Italy). The working standards of benzalkonium chloride (Batch No- B6295) were purchased from Sigma Aldrich (Milan, Italy). Deionized and purified water using a Mili-Q system (Milli-pore) was used for the mobile phase and the standard solutions preparation. All experiment was performed using 'A' class volumetric glassware. All other reagents were of analytical grade.

\section{Chromatographic system}

The liquid chromatograph consisted of a agilent system, equipped with a automatic sample injector and PDA detector. For data collection and calculation chemstation software was used. 
Buffer was prepared by dissolving $3.5 \mathrm{~g}$ of potassium dihydrogen orthophosphate and $3.0 \mathrm{~g}$ of 1 - octane sulphonate in $500 \mathrm{~mL}$ milli-Q water. The $\mathrm{pH} 6.3$ was adjusted with diluted sodium hydroxide solution.

The chromatographic condition was optimized using a column Purospher Star RP-18e $75 \mathrm{~mm} \times 4.0 \mathrm{~mm}, 3.0 \mu$. The mobile phase consisted of acetonitrile: buffer $(65: 35, \mathrm{v} / \mathrm{v})$. The mobile phase was filtered through a $0.22 \mu \mathrm{m}$ nitrocellulose-membrane filter (Milipore, Barcelone) and degassed under vacuum prior to use. The flow rate was $1.8 \mathrm{~mL} / \mathrm{min}$. The monitoring wavelength was $215 \mathrm{~nm}$ and the injection volume was $50 \mu \mathrm{L}$ with maintaining column oven temperature with $25^{\circ} \mathrm{C}$. Peak area was measured and HPLC analysis was conducted at room temperature. Milli-Q water was used as a diluent.

\section{Standard and sample solution preparation}

\section{Standard preparation}

Standard solution of benzalkonium chloride was prepared by using water as a diluent. Standard solution contains $0.1 \%$ of BKC (w/v).

Sample preparation

Ophthalmic solution containing $0.1 \% \mathrm{BKC}(\mathrm{w} / \mathrm{v})$ and $1.0 \%(\mathrm{w} / \mathrm{v})$ sparfloxacin was directly used as such.

\section{System suitability}

Five replicate injections of standard and the system suitability check were done as follows.

1) The $\%$ RSD of the total area of homologues should not more than 2.0

2) The theoretical plate count of $\mathrm{C} 14$ is not less than 1000 and tailing factor is not more than 2.5 .

\section{Validation study}

\section{Specificity / Selectivity}

Specificity of the method was demonstrated by preparing the solutions like mobile phase, diluent, standard, sample placebo solution, placebo spick with API and degradation sample (Figure 2-5). Each solution was injected on to the chromatograph equipped with photo diode array detector. Chromatograms were recorded.

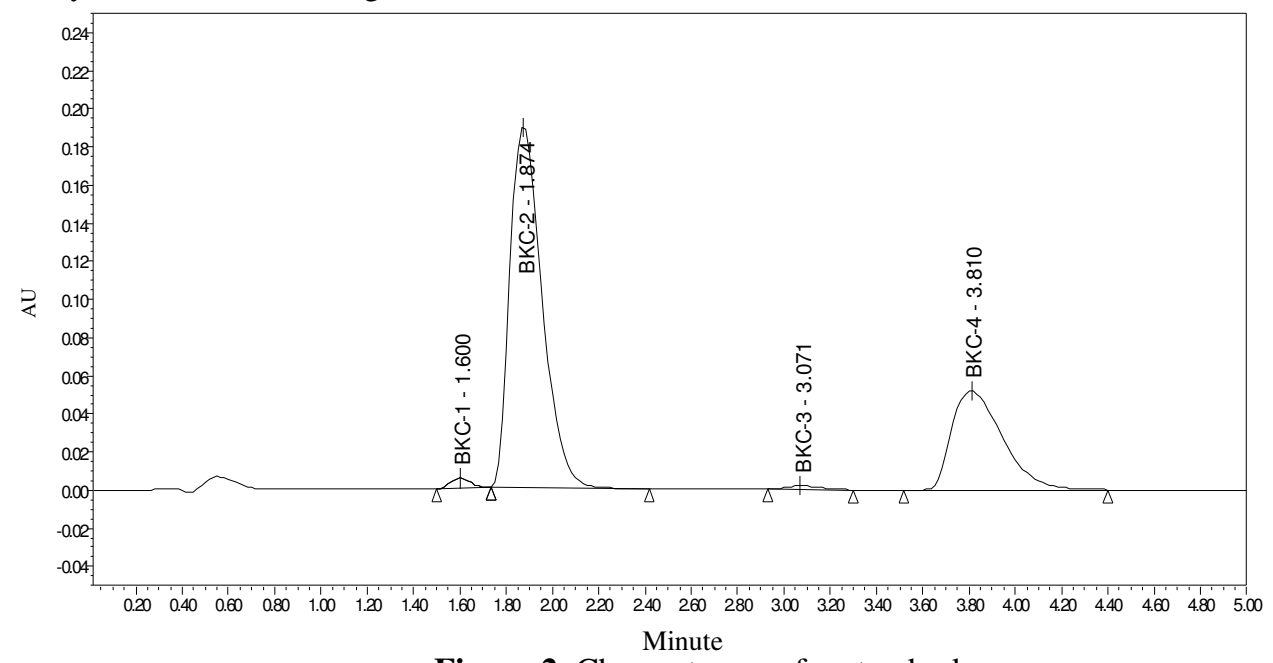

Figure 2. Chromatogram for standard 


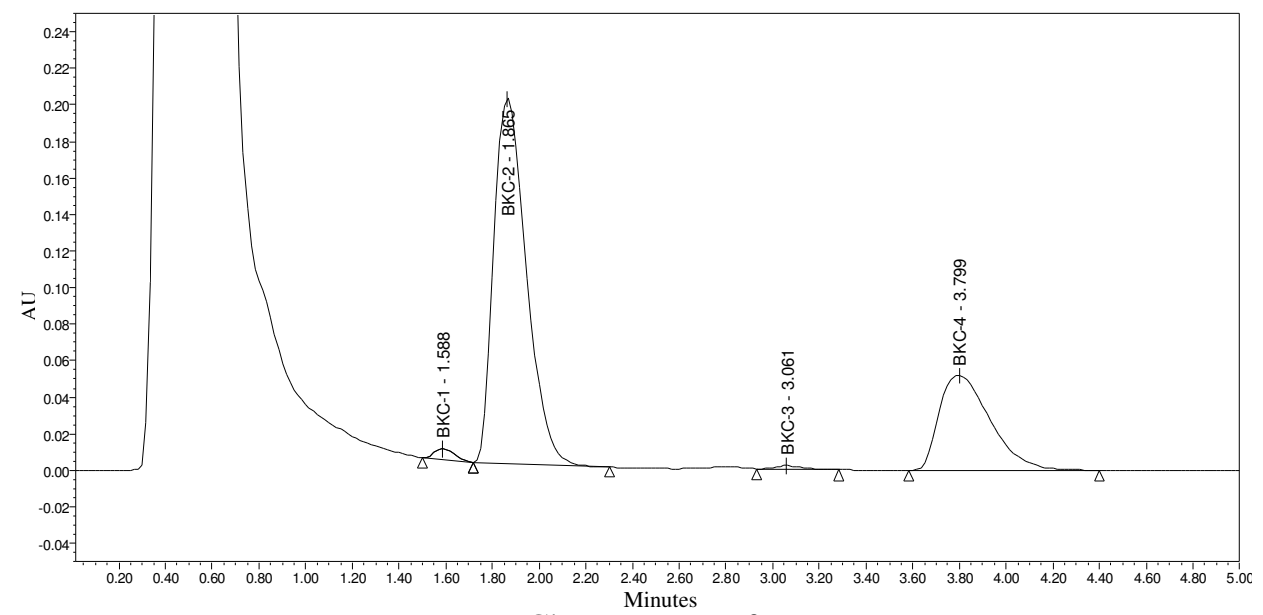

Figure 3. Chromatogram for sample

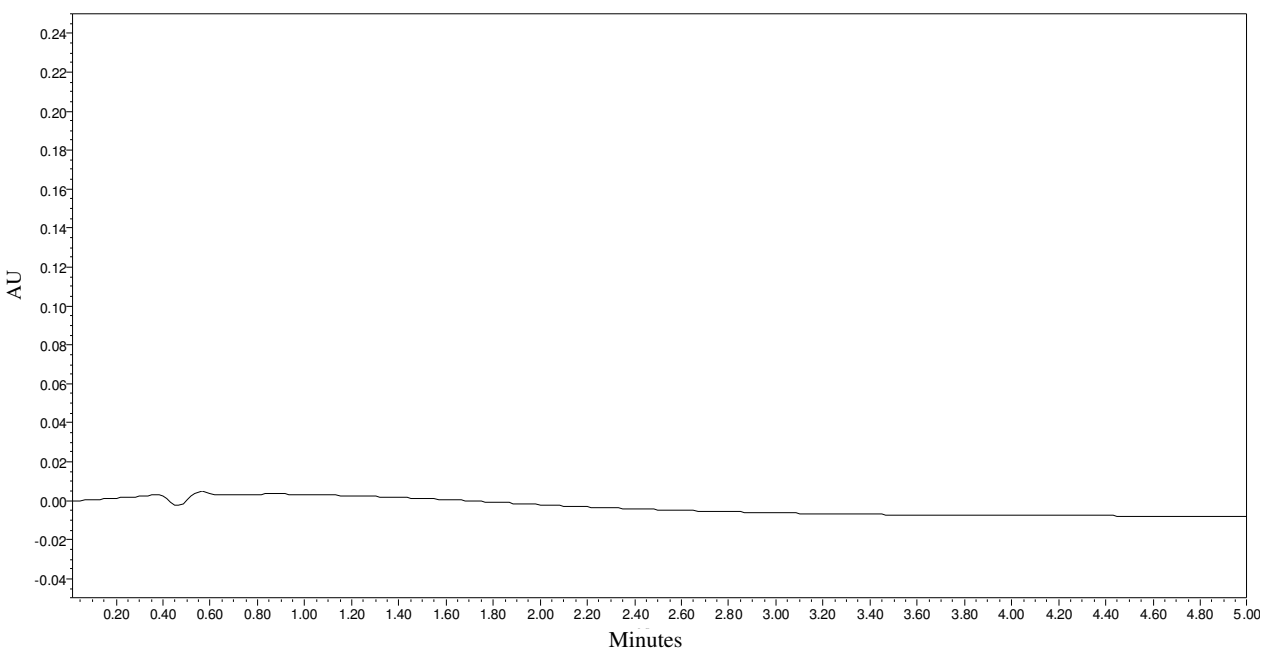

Figure 4. Chromatogram for diluent

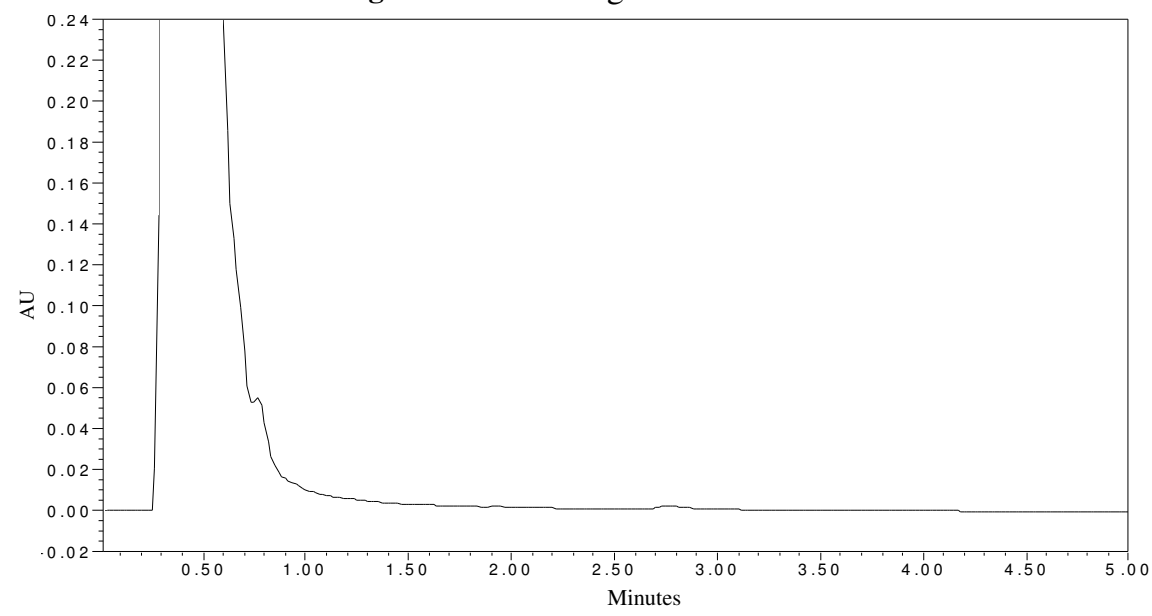

Figure 5. Chromatogram for placebo 


\section{Precision}

The precision is the parameter that expresses the closeness of agreement (degree of scatter) between a series of measurement obtained from multiple analysis of the same homogenous sample under the prescribed conditions. In our study the repeatability was evaluated as follows:

\section{Method precision}

Six consecutive samples were prepared and chromatograms were recorded. The \% assay of benzalkonium chloride was calculated with respect to the standard solutions. The \%RSD of six assay value of sample preparation was less than 2.0.

\section{Intermediate precision}

The aim of the study consists at establishing the effect of the random events on the analytical method the intermediate precision was evaluated by analyzing a sample by different analyst in two different days with different column.

\section{Accuracy (Recovery method)}

Accuracy of a method is defined as the closeness of the measured value to the true value for the sample. The recovery method was studied at concentration levels of $50 \%, 100 \%$ and $150 \%$ of the claimed content, in presence of placebo. Three set for each concentration levels was prepared and injected in duplicate. The recovery was calculated with respect to the standard solutions.

\section{Linearity}

The linearity study verifies that the sample solutions are in a concentration range where analyte response is linearly proportional to the concentration. This study was performed by evaluating the system and method linearity. For the system linearity, standard solutions of benzalkonium chloride at five concentration levels, from 50\%, 75\%, 100\%, 125\% and 150\% of the target analyte concentration were prepared. The concentrations were benzalkonium chloride 50, 75, 100, 125 and $150 \mu \mathrm{g} / \mathrm{mL}$. Each level of concentration was injected in duplicate. The experimental results were plotted to obtain a calibration curve and the corresponding statistical study was performed.

\section{Stability in analytical solution}

The purpose of this experiment is to demonstrate the stability of standard and sample solution used in this method at room temperature (about $25^{\circ} \mathrm{C}$ ). The standard solution and sample solution as given in the methodology was prepared. Both the solutions were injected on to the chromatograph and recorded the chromatograms at $24 \mathrm{~h}$ for standard solution and sample solution. The peak response for the major peak and the percentage deviation in the peak response from initial for both standard and sample solution were evaluated.

\section{Robustness}

Standard solutions of benzalkonium chloride were prepared as per prescribed method and analyzed using different chromatographic condition as below.

(1) Column temperature was changed by $+5^{\circ} \mathrm{C}$ (i.e. $\left.30^{\circ} \mathrm{C}\right)$

(2) The flow rate of mobile phase was changed by \pm 0.2 (i.e. $1.6 \mathrm{~mL} / \mathrm{min}$ and $2.0 \mathrm{~mL} / \mathrm{min}$ )

(3) The wavelength of detector was changed by $\pm 2 \mathrm{~nm}$ (i.e. $213 \mathrm{~nm}$ and $217 \mathrm{~nm}$ )

(4) The $\mathrm{pH}$ of buffer solution was changed by \pm 0.2 unit (i.e. 6.1 and 6.5 )

\section{Results and Discussion}

\section{Method development}

The introduction of new HPLC methods for a routine quality control of pharmaceutical preparations begins with a series of preliminary investigations, which enables establishing 
the optimal experimental conditions and provide maximum relevant information by analyzing the experimental data. In this study, a RP-HPLC method for the determination of benzalkonium chloride was developed and validated. A simple sample preparation, short separation time was considered when the study started.

\section{Observation of Validation study}

\section{Specificity / selectivity}

From the Benzalkonium chloride Standard chromatogram, it was observed that the drug eluted at a retention time of 1.4, 1.6, 2.8 and $3.5 \mathrm{~min}$ for BKC-1, BKC-2(C12), BKC-3 and $\mathrm{BKC}-4(\mathrm{C} 14)$ respectively. The study of the purity of two major responses at $1.6 \mathrm{~min}$ and 3.5 min the peak showed that the five spectrums obtained at different times are within the established threshold for this peak. No interferences with the analyte peaks due to placebo, blank and degradable product have been observed. On the basis of that, the method results specific for the qualitative analysis of benzalkonium chloride.

The peak purity angle should be less than peak purity threshold for waters HPLC system and for Agilent HPLC system peak purity of analyte peak should not be less than 990. It's indicating that all peaks are pure. According to the areas obtained, it can be concluded that all are stable in these conditions. The purity factor for the drug assures that there is no co elution of other peaks. Therefore, the method is specific and suitable for routine work.

\section{Precision}

\section{Instrumental precision (System suitability)}

Five replicate injections of standard preparation and observations are mentioned in the Table 1

Table 1. System suitability observation

$\%$ RSD of area of total homologs. Tailing factor of C14 Theoretical plate count of C14

$$
0.1 \% \quad 2.2 \quad 2124
$$

\section{Method precision}

In this study, a RSD of $1.2 \%$ was observed, by injecting six sets of sample solution. \%RSD for percentage assay results of six sample preparation is than 2.0. The low \%RSD has observed for the six assay result. Hence, it is concluded that method is precise and reproducible for the analysis of said preservative content in this formulation.

\section{Intermediate precision}

The method can be found rugged if the difference between percentage assay results of normal condition and altered condition is not more than 2.0\%. Calculated the percentage assay of each sample and demonstrated the precision by evaluating percentage relative standard deviation of assay results, for which \% RSD observed was $1.2 \%$ and the difference observed between two conditions was $0.3 \%$. Comparison of this results complied the mentioned criteria and method found very much rugged for analysis.

Table 2. Method precision and intermediate precision

\begin{tabular}{ccc}
\hline S. No. & Method precision & Intermediate precision \\
\hline 1 & 98.3 & 98.6 \\
2 & 97.9 & 99.1 \\
3 & 98.0 & 98.5 \\
4 & 95.5 & 98.3 \\
5 & 98.8 & 97.2 \\
6 & 97.1 & 95.9 \\
Mean & 97.6 & 97.9 \\
\% RSD & 1.2 & 1.2 \\
\multicolumn{2}{c}{$\%$ Difference } & $0.3 \%$ \\
\hline
\end{tabular}


Accuracy by recovery

The results obtained for the accuracy study in the samples ranging benzalkonium chloride concentration between $0.05,0.1$ and $0.15 \mathrm{mg} / \mathrm{mL}$ and being the $100 \%$ corresponding to $0.1 \mathrm{mg} / \mathrm{mL}$. ( $\mathrm{n}=3$ for $50 \%, 100 \%$ and $150 \%$ ) indicated that the recovery percent was between 98.8 and $101.8 \%$ of recovery. \% Recovery for the range $50.0 \%$ to $150.0 \%$ of target concentration has found within the acceptance criteria with acceptable \% RSD of not more than $2.0 \%$ at each level. The recovery at each level should be $98.0 \%$ to $102.0 \%$. This indicates that the method is accurate for the analysis of benzalkonium chloride in sparfloxacin eye drops.

Table 3. Accuracy by Recovery

\begin{tabular}{cccc}
\hline S. No. & $\begin{array}{c}\text { \% Recovery of } \\
\text { Benzalkonium chloride }\end{array}$ & $\begin{array}{c}\text { Average of } \\
\text { \% Recovery }\end{array}$ & \% RSD \\
\hline \multirow{2}{*}{1} & $\begin{array}{c}50 \% \text { Recovery set-1 } \\
50 \% \text { Recovery set-1 }\end{array}$ & 100.6 & \multirow{2}{*}{1.1} \\
& $\begin{array}{c}50 \% \text { Recovery set-1 } \\
100 \% \text { Recovery set-1 }\end{array}$ & \\
2 & $100 \%$ Recovery set-1 & 99.1 & 0.3 \\
& $100 \%$ Recovery set-1 & & \\
& $150 \%$ Recovery set-1 & & 0.7 \\
\hline
\end{tabular}

\section{Linearity}

The linearity was determined as linear regression with least square method on standard solution (Figure 2). Concentration levels were 50, 75, 100, 125 and 150\% of the claimed analyte concentration, corresponding to the range of about $50-150 \mathrm{mcg} / \mathrm{mL}$. The calibration curve obtained by plotting the BKC peak area versus the concentration of standard solution was linear in the mentioned concentration range of $50 \%$ to $150 \%$. For acceptance of linearity, correlation coefficient of linearity curve can not be less than 0.9990 and Y-intercept bias should be within $\pm 2.0 \%$ of $100 \%$ linearity response. This indicates that the method is linear up to the specified range of concentrations. Observations were recorded in the Table 4.

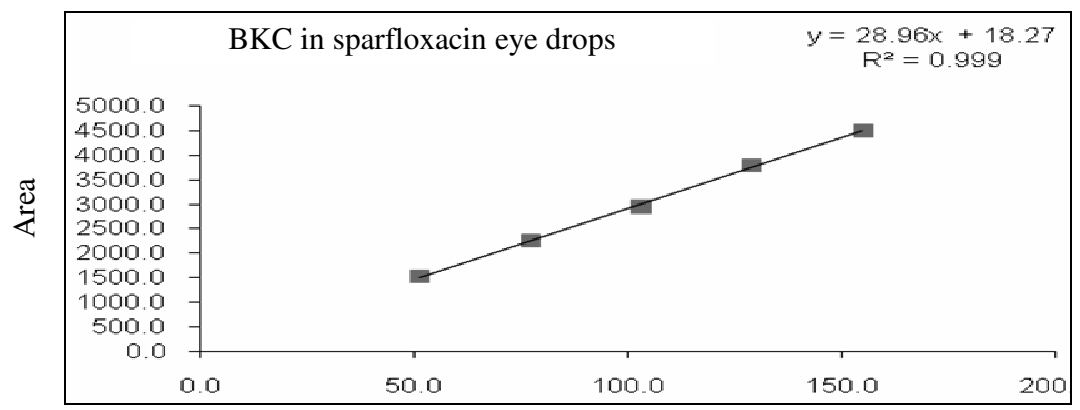

Conc in ppm

Figure 6. Linearity curve of preservative analogue benzalkonium chloride (BKC)

Table 4. Linearity

\begin{tabular}{cc}
\hline Parameter & Benzalkonium chloride \\
\hline Correlation coefficient & 0.999 \\
Slope & 28.96 \\
Y- Intercept bias & $0.6 \%$ \\
\hline
\end{tabular}




\section{Stability of analyte solution}

The solution stability was checked for sample preparation from initial to $24 \mathrm{~h}$. The results obtained are well within the acceptance criteria up to $24 \mathrm{~h}$ at room temperature. Both standard and sample solution thus prepared can be used within this time period.

\section{Robustness}

The quantification was carried out with minor but deliberate changes in the parameters i.e. detection wavelength, column temperature, $\mathrm{pH}$ of mobile phase, change in organic solvent ratio etc. of analytical methodology and system suitability parameters e.g. theoretical plates, tailing, \%RSD were observed and found good with all the altered conditions.

Table 5. Robustness study

\begin{tabular}{lccc}
\hline \multicolumn{1}{c}{ Conditions } & \%RSD of BKC & $\begin{array}{c}\text { Tailing factor of } \\
\text { C14 homolog }\end{array}$ & $\begin{array}{c}\text { Theoretical plate } \\
\text { count of C14 homolog }\end{array}$ \\
\hline Normal condition & 0.4 & 1.6 & 1635 \\
Column temperature, $30^{\circ} \mathrm{C}$ & 0.2 & 1.6 & 1676 \\
Flow rate $1.6 \mathrm{~mL}$ & 0.1 & 1.6 & 1584 \\
Flow rate $2.0 \mathrm{~mL}$ & 0.2 & 1.6 & 1575 \\
Wavelength $213 \mathrm{~nm}$ & 0.8 & 1.6 & 1636 \\
Wavelength $217 \mathrm{~nm}$ & 0.8 & 1.6 & 1636 \\
pH of buffer 6.1 & 0.2 & 1.7 & 1946 \\
pH of buffer 6.5 & 1.0 & 1.7 & 1969 \\
\hline
\end{tabular}

\section{Conclusion}

A reverse phase liquid chromatographic analytical quantification methodology of benzalkonium chloride (BKC) preservative content in pharmaceutical formulation of sparfloxacin ophthalmic solution (eye drop) is developed and validated successfully. This method has significant advantages, in terms of shorter analysis time with excellent resolution, selectivity and accuracy than previously reported. The extraction method gave consistent and reproducible recovery for analyte from formulated preparation, with no interference and matrix suppression. The validation study indicates that method can be considered suitable for carrying out quality control and routine use for quantification of foresaid preservative component with said formulation.

\section{Acknowledgment}

The authors are thankful to M/s Cadila Pharmaceutical Ltd, All raw data form the validation work archived at Cadila Pharmaceutical Ltd. All the validation work performed at Analytical Research Laboratory (ARL), Cadila Pharmaceutical Ltd, Dholka.

\section{References}

1 Shelly J Prince, Hei-Jen McLaury, Loyd V Allen and Phil McLaury, J Pharm Biomed Anal., 1999, 19(6), 877-882.

2 Yuri V. Kazakevich and Rosario LoBrutto, HPLC for Pharmaceutical Scientists, Wiley-Interscience, 2007: ISBN: 0-471-68162-8.

3 Lloyd R. Snyder, Joseph J Kirkland and Joseph L. Glajch, Practical HPLC Method Development, $2^{\text {nd }}$ Edition. Wiley, 1997.

4 Satindr Ahuja and Henrik Rasmussen, HPLC Method Development for Pharmaceuticals, Academic Press, 2007. 
5 Daan J A Crommelin and Robert D Sindelar, Pharmaceutical Biotechnology, Taylor and Francis, 2002, 238-239.

6 Yuri Kazakevich and Rosario Lobrutto, HPLC for Pharmaceutical Scientists, WileyInterscience, 2007.

7 Ya-Hui Hou, Chien-Yi Wu and Wang-Hsien Ding, J Chromatogr A., 2002, 976(1-2), 207-213.

8 Kibbe A H, Handbook of Pharmaceutical Excipients, $3^{\text {rd }}$ Ed., American Pharmaceutical Association, Washington D C, Pharmaceutical Press, London. 


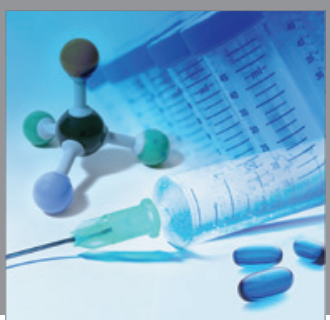

International Journal of

Medicinal Chemistry

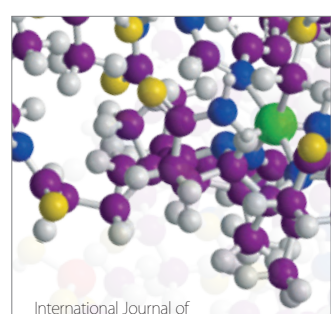

Carbohydrate Chemistry

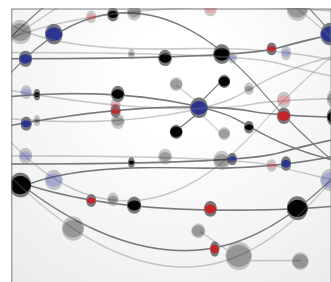

The Scientific World Journal
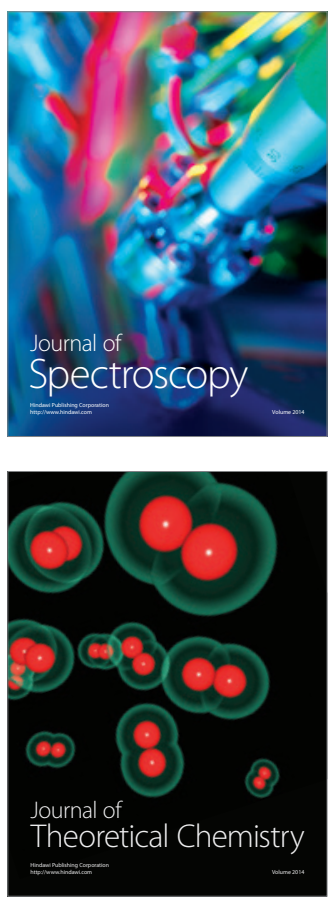
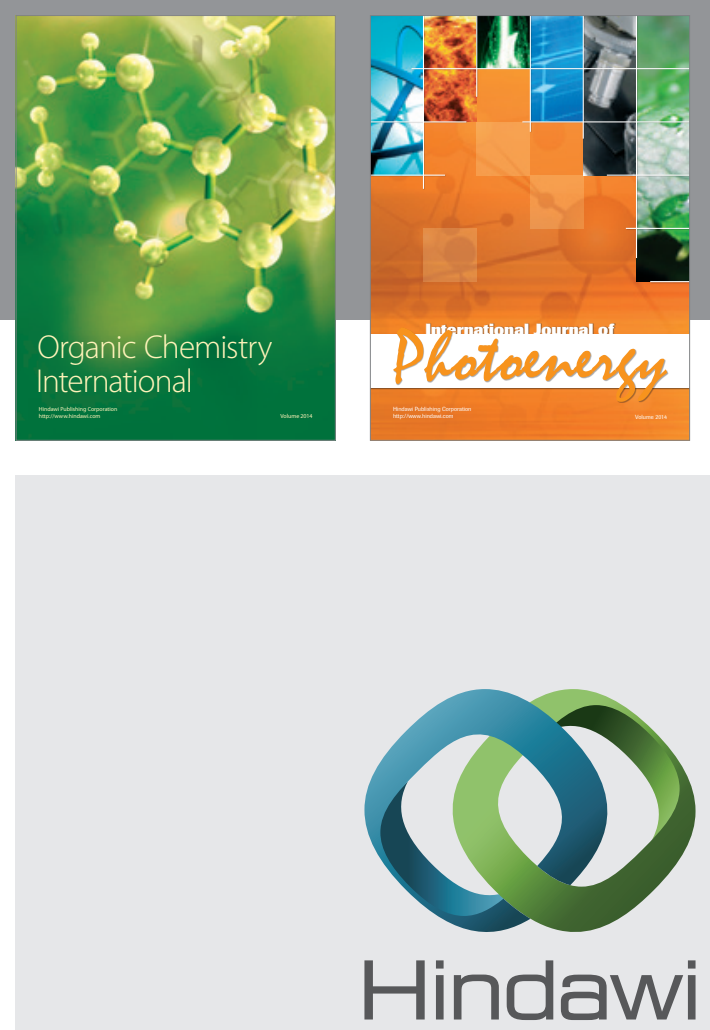

Submit your manuscripts at

http://www.hindawi.com
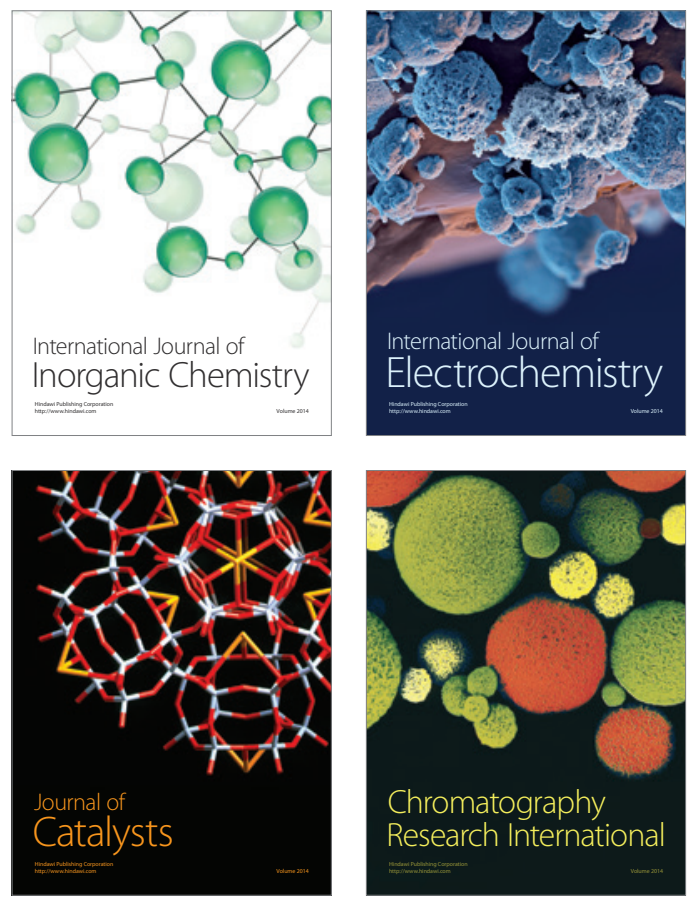
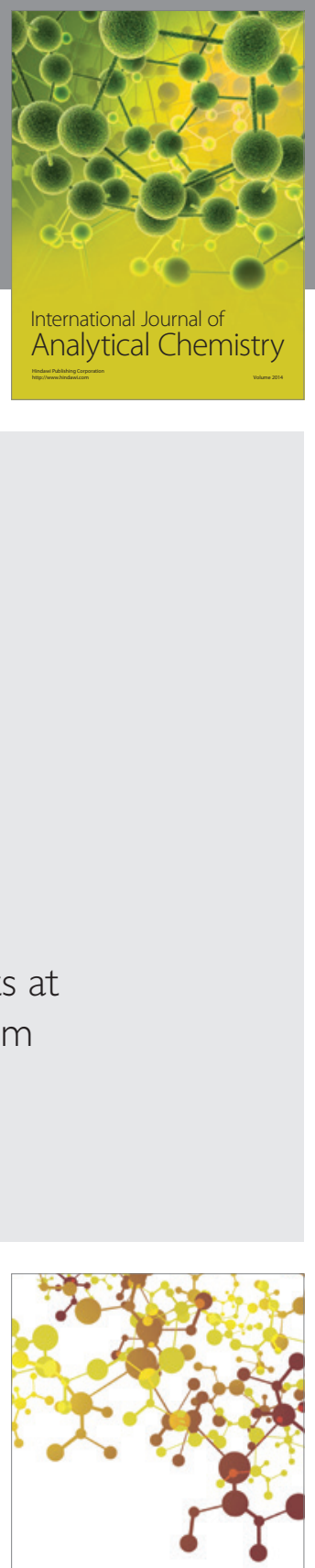

Journal of

Applied Chemistry
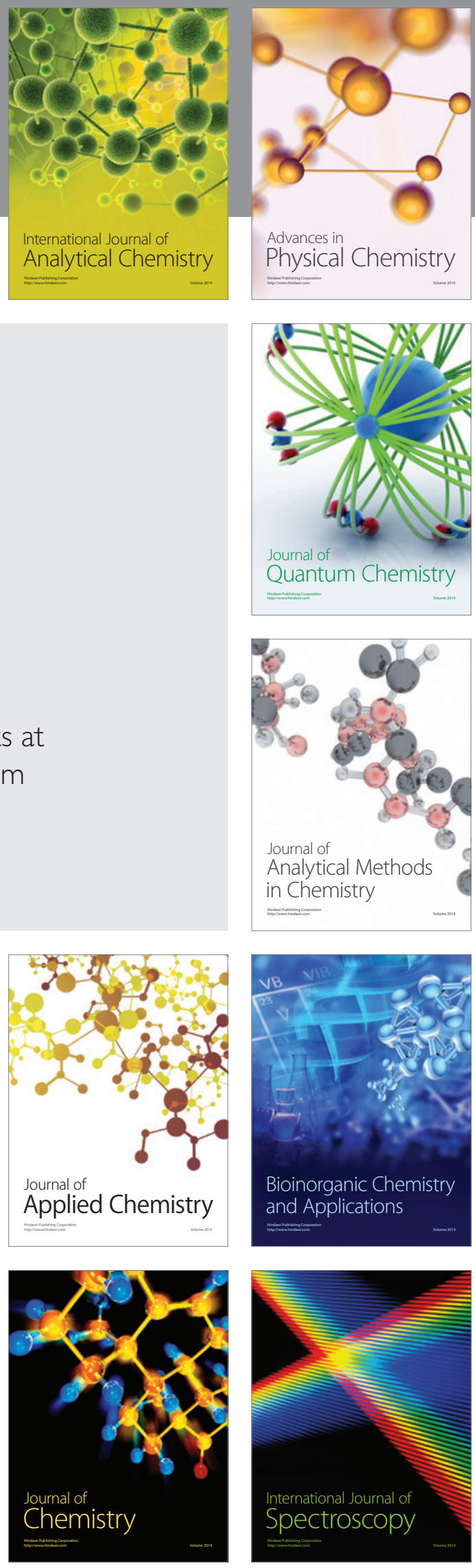